\title{
Designing and Modeling MEMS Filter for Mobiles Transceivers
}

\author{
M. Al_Khusheiny \& B. Y. Majlis,senior member IEEE, \\ Silicon Valley for Nanotechnolgy \\ Riyadh ,Saudi Arabia \\ Email:info@ssv.com.sa
}

\begin{abstract}
In this paper a 71-MHz band-pass IF MEMS filter with bandwidth of $140 \mathrm{KHz}$ is designed, modeled and simulated, the supposed MEMS filter is comprised of two symmetrical Clamped-Clamped $\mu$ resonators, coupled by soft mechanical beam (spring), the centre frequency of this filter is the same of the resonance frequency of the constituent $\mu$ resonator. The bandwidth is determined by the physical dimensions of the coupling beam and its location between the two presonator beams. The center frequency of the supposed filter is $71 \mathrm{MHz}$ where the $Q$ factor is 511 and insertion loss of $7.8 \mathrm{~dB}$, this device can be direct placed in the front-end wireless transceiver circuit.
\end{abstract}

\section{Introduction}

Heterodyning transceivers demand a high- band-pass filters in the radio frequency (RF) and intermediate frequency (IF) , and these are realized using off-chip, mechanically resonant components, such as crystal filters and surface acoustic wave (SAW) devices, due to its high quality factor .Recently due to the relative ease and large stiffness-to-mass ratios, Clamped-Clamped beam (CCbeam) $\mu$ mechanical resonators have been intensively investigated for VHF range applications [2,3]. A CCR $\mu$ resonator was demonstrated within $f_{o}$ of $8 \mathrm{MHz}$ with By suppressing anchor losses, high-stiffness VHF resonator beams can now be utilized with dynamic ranges more applicable to communications applications; so using this technique, free-free beam $\mu$ mechanical resonators were demonstrated with center frequencies from 30 to 90 $\mathrm{MHz}$, stiffness from 30000 to $80000 \mathrm{~N} / \mathrm{m}$, and $Q$ 's as high as 8400 [1].

In this paper a device comprised of interlinked micromechanical $\mu$ resonators has been demonstrated to perform highly selective low-loss filtering of applied electrical input signals. In particular, successful subsequent high- bandpass filtering at the VHF are demonstrated using this single, passive, and micromechanical device, all with less than $7.8 \mathrm{~dB}$ of filtering insertion loss. The supposed MEMS filter is composed of two symmetrical Clamped-Clamped $\mu$ resonator (CCR) of resonance frequency $\left(f_{o}\right)$ of 71 $\mathrm{MHZ}$, coupled by soft mechanical beam (spring), the centre frequency of this filter is the same of the resonance frequency of the constituent $\mu$ resonator .while the bandwidth is determined by the physical dimensions of the coupling beam and its location between the two $\mu$ resonator beams. As the location of the coupling is nearer to the anchor of the constituent $\mu$ resonators as the filter Quality factor is being larger and the bandwidth is decreased.

The center frequency of the supposed filter is $71 \mathrm{MHz}$ where the Q-factor is 511 with bandwidth of $140 \mathrm{KHz}$ which is $0.2 \%$ of the resonance frequency. And this device can be used directly in the transceiver circuit as we will see later.

\section{MEMS Filter Operation}

Figure 1 presents a perspective view of the MEMS filter, identifying key components. As shown, the filter consists of two polysilicon $\mu$ mechanical clampedclamped beam resonators, coupled mechanically by soft springs. Polysilicon electrodes underlie the central regions of each resonator, and serve as capacitive transducer electrodes positioned to induce resonator vibration in a direction perpendicular to the substrate. The gap between the beam and the electrode is $0.05 \mu \mathrm{m}$.

Under normal operation, the device is excited capacitively by a signal voltage $V_{p}$ applied to the beams

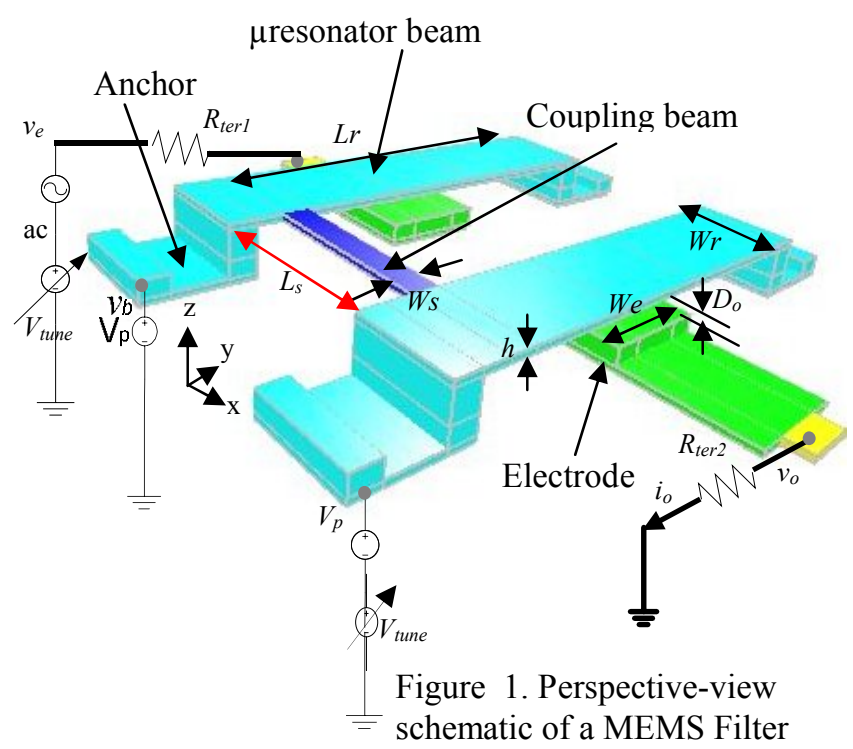

of the two resonators, and another ac signal excited to the input electrode of the resonator-1(port-1). The output is 
taken at the other port of the structure (resonator-2), also via capacitive transduction. . The application of this input creates a directed electrostatic force between electrode-1 and the conductive resonator beam that induces z-directed vibration of the input resonator. When the frequency of the ac-input voltage comes within the passband of the mechanical filter; this vibrational energy is transferred to the output resonator by the coupling spring, causing the second beam vibrating.

The z-directed force produced between the input electrode and the beam of the first constituent resonator of the filter, can be expressed as the derivative of the energy $E$ stored in the electrode-to-resonator capacitor with respect to resonator beam displacement, and this force can be expressed as:

$$
\begin{aligned}
& F=\frac{\partial C}{\partial z}\left(V_{P}^{2} / 2+V_{i}^{2} / 4\right)-V_{P} \frac{\partial C}{\partial z} V_{i} \cos \omega_{i} t+ \\
& \frac{\partial C}{\partial z}\left(V_{i} / 4\right) \cos 2 \omega_{i} t
\end{aligned}
$$

where $v_{i}=v_{b}-v_{e}$, as shown in Figure 1, is the difference voltage between the beam and electrode, while an $a c$ excitation signal $v_{e}=V_{i} \operatorname{Cos}\left(\omega_{i} t\right)$ is applied to the underlying electrode.

The first term in (1) represents the dc force which statically bends the beam. While the second term constitutes a force at the frequency of the input signal, amplified by the dc-bias voltage $V_{p}$, which is the main input component used in tank and filter applications. When $\omega_{i}=\omega_{o}$ (the resonance frequency) this force drives the beam into resonance, creating a dc-biased (via $V_{p}$ ) time-varying capacitance between the electrode and resonator beam, and producing an output current $i_{o}$.

The magnitude of electromechanical coupling achieved at the input and output transducers is proportional to the square of the electrode-to resonator overlap capacitance, and to the square of the dc-bias voltage $V_{p}$ shown applied to the conductive resonator structure ,as shown in the equation( 1 ) .

Thus, to get a good electromechanical coupling, the capacitor gap spacing should be minimized, and the voltage $V_{p}$ should be as large as permissible. Under applying an input excitation voltage with suitable frequency, the constituent resonator begins to vibrate in one or more flexural modes in a direction perpendicular to the substrate. For a properly designed micromechanical filter, if the excitation voltage has a frequency within the pass-band, both resonators will vibrate. The vibration of the output resonator then imports to the output electrode, providing an output current given by:

$$
i_{o}=V_{D C}\left(\partial C_{2} / \partial z\right)(\partial z / \partial t)
$$

where $\mathrm{z}$ is vertical displacement, $V_{D C}=V_{p}-V_{n}$, with $V_{n}$ equal to the $r m s$ value of the ac signal at electrode $n$, and $\partial C_{2} / \partial z$ is the change in resonator-to-electrode capacitance per unit displacement at $\mu$ resonator 2 . The current $i_{o}$ is then directed to resistor $R_{\text {ter } 2}$, which provides the proper termination impedance for the $\mu$ mechanical filter. $R_{\text {ter } 2}$ then feeds a transresistance amplifier which amplifies $i_{o}$ to a buffered output voltage $v_{o}$, as shown in figure 1.As a result, what's happening is that the device takes an electrical input signal converting it to mechanical movement of the first beam, and this movement imported to the other beam by the coupling beam ,then reconverted to electrical output signal from the second electrode.

\section{MEMS Filter Design}

The force which results by applying the voltage between the beam and electrode will be as follow:

$$
F=V_{D C} \frac{\partial C_{n}}{\partial z} v_{i}, \text { and } \frac{\partial C_{n}}{\partial z} \cong \frac{W_{r} W_{e}}{D_{o}^{2}}
$$

where the $\partial C_{n} / \partial \mathrm{z}$ is the change in resonator-to-electrode capacitance per unit displacement at $\mu$ resonator $n$, and $W_{e}$ and $W_{r}$ are electrode width and beam width respectively shown in Fig 1 , and $D_{o}$ is the electrode-toresonator gap.

The effective resonator spring stiffness $k_{r}(y)$ must be maximized, while its effective mass $m_{r}(y)$ is minimized. The $\mu$ resonators comprising the filter are preferably designed to be identical, each with the same resonance frequency, given by (neglecting stress and width effects) [4]

$$
f_{o}=f_{n o m}\left[1-\left\langle\frac{k_{e}}{k_{m}}\right\rangle\right]^{\frac{1}{2}}
$$

and

$$
\left\langle\frac{k_{e}}{k_{m}}\right\rangle=\int_{L_{1}}^{L_{2}} \frac{d k_{e}\left(y^{\prime}\right)}{k_{m}\left(y^{\prime}\right)}=\int_{L 1}^{L 2} \frac{\left(V_{p}^{2} \varepsilon_{o} W_{r}\right.}{[d(y)]^{3} k_{m}(y)} d y
$$

where $\quad L_{1}=0.5\left(L_{r}-W_{e}\right)$, and $L_{2}=0.5\left(L_{r}+W_{e}\right)$, and $d(y)$ represent the deviation of the beam [2].

To calculate the nominal resonance frequency of the resonator $f_{\text {nom }}$ (without applying any voltage ) Timoshenko method had been used, which can be calculated by solving the following equations which are shown below:

$$
\begin{aligned}
& \tan \frac{\beta}{2}+\frac{\beta}{\alpha}\left(\frac{\alpha^{2}+g^{2}(k G / E)}{\beta^{2}-g^{2}(k G / E)}\right) \tanh (\alpha / 2)=0, \\
& \left.\begin{array}{l}
\alpha^{2} \\
\beta^{2}
\end{array}\right\}=\frac{g^{2}}{2}\left[\mp\left(1+\frac{E}{k G}\right)+\sqrt{\left(1-\frac{E}{k G}\right)^{2}+\frac{4 L_{r}^{2} h W_{r}}{g^{2} I_{r}}}\right]
\end{aligned}
$$




$$
\begin{aligned}
& I_{r}=\frac{W_{r} h^{3}}{12} \quad \text {, and } \quad G=\frac{E}{2(1+v)} \\
& g^{2}=\left[2 \pi f_{\text {nom }}\right]^{2} L_{r}^{2}\left(\frac{\rho}{E}\right)
\end{aligned}
$$

where $h$ is the beam thickness, $k_{r}$ is the dynamic spring constant of the $\mu$ resonator, $m_{r}$ is its effective mass, and $E$ and $\rho$. are the Young's modulus and density of the structural material, respectively. Equation (4) suggests that, high frequency resonators require high spring stiffness and low mass.

When each resonator is designed to have the same resonance frequency $f_{o}$, as a result the passband of the overall filter will be centered around this frequency $f_{o}$. The coupling spring, acts to pull the resonator frequencies apart, creating two closely spaced resonance modes that constitute the filter passband. Thus, the center frequency of a mechanical bandpass filter is determined by the geometry of the constituent resonators, while its bandwidth is determined by the coupling spring (dimensions and its location on the CCR's Beams). In order to design the resonator and coupling spring topologies properly, electromechanical analysis is needed, where the electrical domain inductance and capacitance of a properly synthesized LC ladder filter are implemented via analogous values of compliance and mass in the mechanical domain.

\subsection{The Equivalent Circuit in Mechanical \& Electrical Domain}

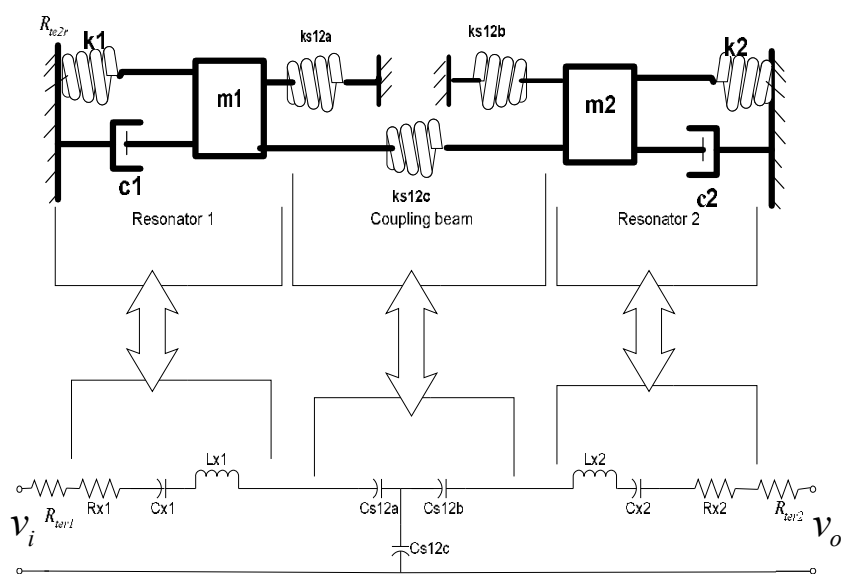

Figure 2. The equivalent circuit of the MEMS Filter in mechanical and electrical domain

In order to design the filter, an electrical equivalent circuit is needed [5,6]. As shown in figure 2, both electrical and mechanical inputs and outputs are possible for this device, so the equivalent circuit must be able to model the two domains. In addition, a circuit model that directly uses the lumped mechanical elements summarized by (7)-(9) is preferred. Figure 2 presents the equivalent circuit used in this work, which is modeled by
LCR circuit with element values corresponding to actual values of mass, stiffness, and damping. The damping factor of the beam is calculated from the following equation:

$$
D_{r}(y)=\frac{\sqrt{k_{m}(y) m_{r}(y)}}{Q_{\text {nom }}}
$$

The dynamic stiffness of the resonator is: $k_{r}(\mathrm{y})=$ $k_{m}(\mathrm{y})-k_{e}(\mathrm{y})$, where $k_{e}(y)$ is the electrical spring constant, ,and $k_{m}(y)$ is the mechanical spring constant of the resonator without applying any external force on it.

Practically the transformed LCR circuit of the filters elements value are given in the next equations:

$$
L_{x}=\frac{m_{r e}}{\eta^{2}}, \quad C_{x}=\frac{\eta^{2}}{k_{r e}}, \quad R_{x}=\frac{D_{r e}}{\eta^{2}}
$$

The subscript $e$ denotes the electrode location at $y$ $=L r / 2$, and $R_{x}$ is the motional resistance seen a cross the electrical -to-resonator gap at resonance.

The electromechanical transformer turns ratio $\eta$ can be obtained via an impedance analysis, which is :

$$
\eta=V_{D C} \frac{\partial C_{n}}{\partial z}
$$

Where $\partial C_{n} / \partial \mathrm{z}$ is given before in eq.(4) .

\subsection{Coupling Beam Design}

In order to attach the two resonator together, a nondoped polysilicon coupling beam should be used .For a given filter center frequency $f_{o}$ and bandwidth $B W$, the required coupling beam spring constant can be found using the expression [2]:

$$
k_{s 12}=k_{r} \frac{B W}{f_{o}} k_{12}
$$

Where $k_{12}$ is the normalized coupling coefficient between resonator tanks for a given filter type [6]. The required value of coupling spring constant $k_{s 12}$ is determined mainly by the coupling beam geometry, and is given by the expression [7]:

$$
k_{s 12}=\frac{E I \psi^{3}(\sin \psi+\sinh \psi)}{L_{12}^{3}(\cos \psi \cosh \psi-1)}
$$

Where $\psi=L_{12}\left(\rho A \omega^{2} / E / I_{r}\right)^{0.25}$, and $I_{r}$ is shown in (6). The design of the coupling beam is complicated by the fact that the beam itself has finite mass. In particular, for the case of micro-scale filters, the coupling beam mass is on the same order as that of the resonator beams. In this 
case the beam mass can added to the masses of the adjacent resonators, thereby changing their individual resonance frequencies, and in turn, changing the overall filter center frequency. From an equivalent circuit viewpoint, the coupling beam can in general be represented by a T-element composed of three capacitors, as shown in figure 2. The shunt capacitor $C_{s 12}$ represents the desired spring action of the coupling beam, $C_{s 12 a}=\eta^{2} / k_{s 12}$; where $\eta$ is given in eq.(9). The series arm capacitors $C_{s 12 a}$, however, represent the finite mass of the coupling spring, and add to the LCR's of adjacent resonators, effectively changing their frequencies. To eliminate this phenomenon, the coupling spring must be designed so that $C_{s 12 a}=C_{s 12 b}=-C_{s 12 c}$. Under this condition, the effects of the series arm capacitors are cancelled to first order during filter operation, making the coupling beam appear massless. $C_{s 12 a}=C_{s 12 b}=-C_{s 12 c}$ is satisfied when the coupling beam length $L_{12}$ corresponds to a quarter wavelength at the frequency of operation; i.e., when $L_{12}$ satisfies the expression [7]:

$$
\cos \psi \operatorname{sonh} \psi+\sin \psi \cosh \psi=0
$$

After determination of $L_{12}$ via (12), $W_{12}$ is chosen to satisfy (10) and (11).

\subsection{Coupling Beam Location \& Filter Bandwidth}

The filter quality factor $\left(Q_{\text {filter }}=f_{o} / B W\right)$ attainable via a mechanical filter is proportional to the ratio of the resonator and coupling beam spring constants $k_{r} / k_{s I}$ [2]. For macroscopic mechanical filters this ratio can be made quite large, because the resonators are often much bigger and thicker than their associated coupling springs. On the other hand, in $\mu$ mechanical filters, the resonators and couplers are usually of similar size, and thus, the ratio $k_{r} / k_{s 12}$ is limited, thus, the attainable $Q_{\text {filter }}$ is limited. A novel method for attaining greater $Q_{\text {filter }}$ takes advantage of the fact that the dynamic spring constant $k_{r}$ of a clamped-clamped beam is larger at locations closer to the anchor points-i.e., it's larger at points moving with lower velocity at resonance. Thus, by coupling the beams closer to the anchor points, rather than at the centers of resonator beams, higher $Q_{\text {filter }}$ can be achieved, even when the resonators and coupling springs have similar sizes.

\subsection{Filter Termination and Insertion Loss}

The terminating resistors $R_{\text {ter }}$ represent the resistance of the input (source) and output (load) circuits. It is necessary to terminate the MEMS filter with resistance in order to flatten the passband of the filter. Without proper termination, the resonator Q's are too large, and the filter passband consists of distinct peaks of selectivity; and in order to flatten the passband, the Q's of the constituent resonators must be reduced, by terminating the filter with the proper resistors.

In figure 2 , resistors $R_{\text {ter } 1}$ and $R_{\text {ter } 2}$ represent the termination resistors that decrease the effective $\mathrm{Q}$ of the constituent resonators. The needed value of termination resistance for a $\mu$ mechanical filter with center frequency $f_{o}$ and bandwidth $B W$ should be equaled at the two sides (input and output, [5]), and can be expressed as:

$$
R_{Q 1}=R_{Q 2}=\left(\frac{Q_{\text {nom }}}{Q_{\text {filter }}}-1\right) R_{x 1}
$$

where $Q_{n o m}$ is the uncontrolled quality factor of the constituent resonator, and $Q_{\text {filter }}=f_{o} / B W$.

To determine the efficiency of the filter, and its ability to be used in the new microdevices, it important to calculate its insertion loss [8]. The insertion loss of MEMS filter is controlled by the $\mathrm{Q}$ of the individual resonators, and the termination resistance added to flatten the passband, and can be expressed as follow:

$$
I L_{\text {filter }}=20 \log \left(\frac{2 R_{\text {ter } 2}+n R_{x}}{2 R_{\text {ter } 2}}\right)
$$

where $n$ is the order of the MEMS filter, as shown in eq (13), the value of termination resistance depends on both the Bandwidth of the filter and the $\mathrm{Q}$ of the individual $\mu$ resonators .

\section{Results and Analysis}

The work of this paper is processed as follows:

- Designing the two symmetrical CCRs with resonance frequency of $71 \mathrm{MHz}$.

- Designing the coupling beam.

- Choosing the location of the coupling beam to determine the bandwidth and quality factor of the filter.

- Generating the complete electrical equivalent circuit of the filter to simulate it by PsPice or other simulator to verify the design.

Micromechanical CCR's are modeled and designed as detailed in [9], using a polysilicon surface micromachining technology,. IntelliFab software was used to design the fabrication process of the proposed device.

\subsection{Designing MEMS Filter and Results Analysis}

A two symmetrical CCR's had been designed and non-doped coupling beam (spring), to import the vibration between the CCR beams under excitation, was designed also; which was designed to seem massles, as described in section 3.2. The location of the coupling beam was accurately choosed to achieve an over all 
quality factor for the filter of 511 under vacuum, and a bandwidth of $140 \mathrm{KHz}$.The value of the termination resistance was properly determined to flatten the passband of the filter. Figures 1,2 show the perspective view of the MEMS filter with its equivalent circuit in the electrical and mechanical mode respectively, as shown in figure 2 , the coupling beam can be electrically modeled at the lowest order of approximation as a shunt capacitance as explained in section 3.2.

Table 1 shows the MEMS filter data performance summery. In general the center frequency of the filter was determined by the resonance frequency of the constituent $\mu$ resonator, and the shape factor of the filter is determined by the number of the resonators which was cascaded, while the bandwidth of the filter was determined by the mass of the coupling beam and its coupling point's location.

Table 1. MEMS Filter Performance Summery

\begin{tabular}{|c|c|c|c|}
\hline \multirow{2}{*}{ parameter } & \multicolumn{2}{|c|}{ value } & \multirow{2}{*}{ unit } \\
\cline { 2 - 3 } & Calculated & $\begin{array}{c}\text { Simulated } / \\
\text { intellsuitte }\end{array}$ & \\
\hline $\begin{array}{c}\text { Resonator Mass } \\
m_{r e}(y=L r / 2)\end{array}$ & 0.23 & & $p g$ \\
\hline $\begin{array}{c}\text { The resonance } \\
\text { frequency } f_{o}\end{array}$ & 71.5 & 71 & $\mathrm{MHz}$ \\
\hline $\begin{array}{c}\text { The applied dc } \\
\text { voltage } V_{p}\end{array}$ & 30 & 30 & volts \\
\hline $\begin{array}{c}\text { The Pull-In } \\
\text { Voltage } V_{P I}\end{array}$ & 132.5 & 129 & Volts \\
\hline $\begin{array}{c}\text { MEMS Filter } \\
\text { Quality Factor, } Q\end{array}$ & 511 & & $\ldots \ldots$ \\
\hline$L_{c}$ (from anchor) & 2.6 & & $\mu m$ \\
\hline$L_{s}$ & 8.1 & & $\mu m$ \\
\hline$R_{Q}$ & 5.2 & & $\mathrm{k} \Omega$ \\
\hline$I L$ & 7.8 & & $\mathrm{~dB}$ \\
\hline Bwidth & 140 & & $\mathrm{KHz}$ \\
\hline Percentage $B W$ & $0.2 \%$ & & \\
\hline Ws & 1 & & $\mu m$ \\
\hline
\end{tabular}

The filter which was introduced is characterized by a very high selectivity under vacuum conditions, with quality factor of 511 for the filter and 4500 for the constituent $\mu$ resonator. Thus, the filter excited by the

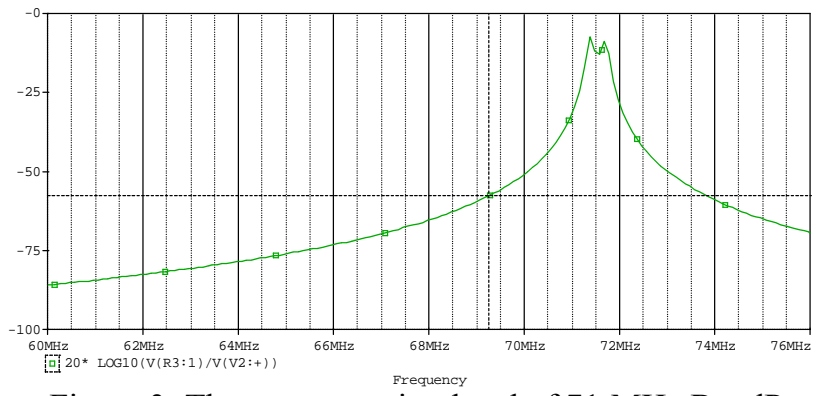

Figure 3. The spectrum simulated of $71 \mathrm{MHz}$ BandPass MEMS filter. $\mathrm{Q}=511$, and $\mathrm{IL}=7.8 \mathrm{~dB}$.

mechanical vibration will oscillate around its own typical frequencies (different modes), but by choosing the geometrical and physical properties of the filter materials, beside the excitation voltage, the output will be determined with a certain frequency.

Figure 3 is the spectrum simulated of MEMS filter, the centre frequency is $71 \mathrm{MHz}$, with $\mathrm{Q}$ of 511 under vacuum, and from the figure also we can calculate the 20-dB-shape-factor which is 2 in the proposed design.

The filter bandwidth is a function of the equivalent mass of the resonator and the compliance of the coupling spring as shown in eq (10). The bandwidth increases as either the mass of resonator or the compliance of the coupling spring decrease. The amplitude versus frequency shape of the passband is related to the variation of the coupling between the two resonators; greater coupling between the resonators ,results in less passband ripple and eventually a rounded passband shape as shown in the figure 3 .

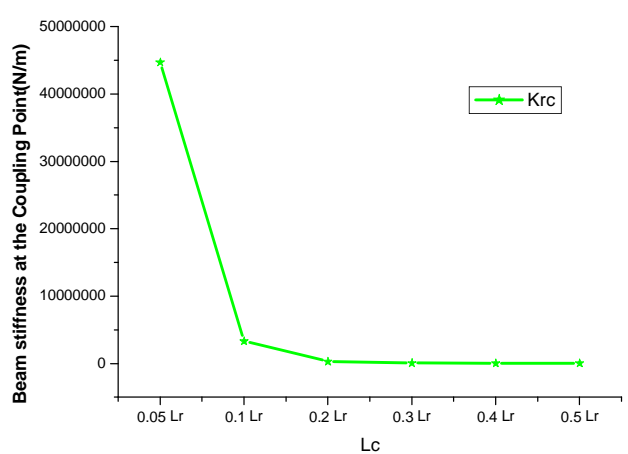

Figure 4. Plot of theoretical Beam stiffness at the coupling location along the beam length.

Figure 4 illustrate simulation result of the beam stiffness with respect to the coupling location along the beam length, as shown in the figure, the beam stiffness at the coupling location is increased with being nearer to the anchor of the constituent beam, and this is return to the fact that, the velocity of the beam at the resonance will be decreased as we are going toward the anchor.

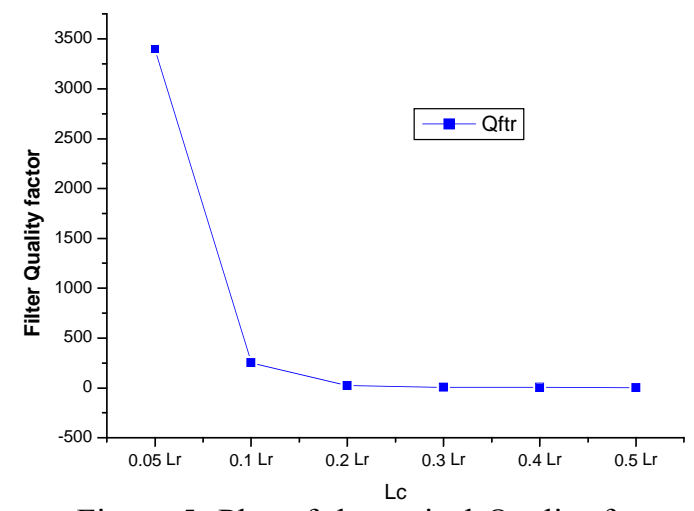

Figure 5. Plot of theoretical Quality factor of the MEMS filter with respect to the coupling location along the beam length.

Figure 5 illustrate simulation results of MEMS filter at $71 \mathrm{MHz}$ with different quality factors, showing the dependent of the quality factor on the location of the coupling with respect to anchor of constituent resonator ,as the coupling location is going toward the anchor the filter quality factor increases, other wise the $\mathrm{Q}$ of the 
filter decreases until reach its minimum value at the mid of the beam (i.e. $y=L_{r} / 2$ ).In our design the coupling location was at $1.2 \mu \mathrm{m}$ from the anchor of the constituent resonators, while the coupling beam length was $8.1 \mu \mathrm{m}$.

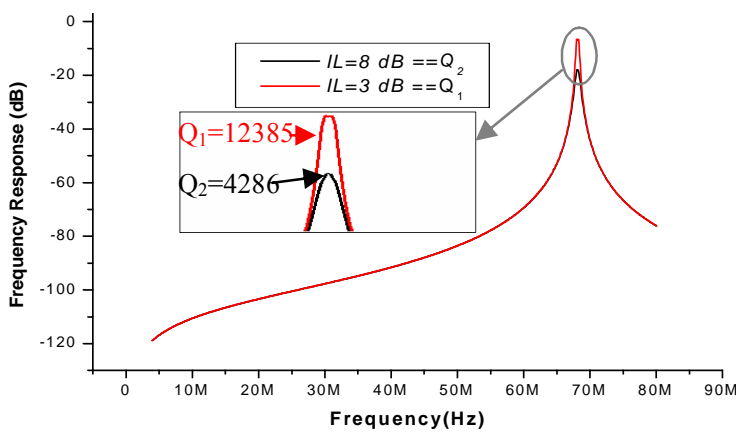

Figure 6. The simulation results of the MEMS filter with different resonator quality factor (Q)[8].

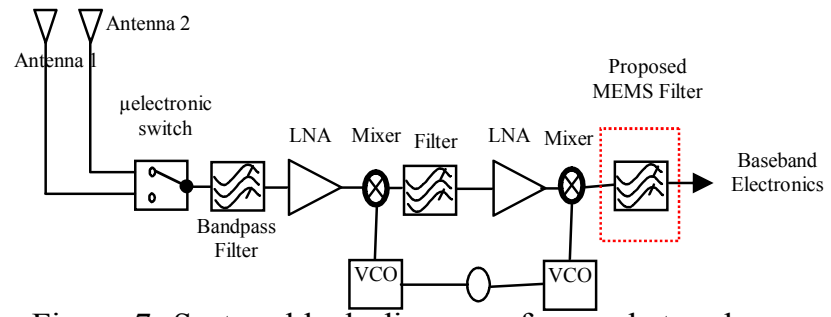

Figure 7. System block diagram of super-heterodyne receiver architecture, showing potential replacement via MEMS-base components.

The insertion loss of the MEMS filter is determined by the $\mathrm{Q}$ of the individual $\mu$ resonators, the order of the filter ,and the termination resistors added to flatten the passband as shown in eq (14). Figure 6 shows the simulation results of the MEMS filter with different resonator quality factor (Q), showing the dependence of the insertion loss on the $\mathrm{Q}$ of individual resonators. As the Q`s are higher results in decreasing the insertion loss.

The total area of the proposed device is $(40 \times 40 \mu \mathrm{m} 2)$, so its smaller and lighter in weight than the traditional filters (i.e. area of several $\mathrm{mm}^{2}$ ) and because the miniaturization offers many advantages, such as enhanced mobility, reduced cost and improved form factors. Thus, one of the primaries focuses for research and development in the area of radio frequency (RF) wireless communications electronics has been device miniaturization. the proposed device has a lighter weight ,good stability with temperature ,small size, low power consumption, very high precision in manufacture, and the potential for low cost through batch fabrication. For these reasons this device can be placed in the front-end transceivers. Figure 7 shows the simplified block diagram of a wireless receiver, indicating (with shading) the components replaceable by the proposed MEMS filter.
A MEMS filter design with centre frequency of 71 $\mathrm{MHz}, Q$ of 511 , and $I L$ of $7.8 \mathrm{~dB}$, based on mechanical coupling of two symmetrical CCR's was introduced. The parameters which affect the overall quality factor of the MEMS filter were discussed.

Simulated results of the MEMS filter show high $Q$ quality factor as the coupling location is nearer to the anchor of the constituent resonators.

For its small size, and the ability to save more power; the proposed device is very suitable to be placed in the circuit of the front-end wireless transceivers

\section{References}

[1] A.-C. Wong, J. R. Clark, and C. T.-C. Nguyen, "Annealactivated, tuneable, $65 \mathrm{MHz}$ micromechanical filters" Digest of Technical, Papers, 10th International Conference on Solid-State Sensors and Actuators, Sendai, Japan, June 7-10, 1999, pp. 1390-1393.

[2] Frank D. Bannon, III, John R. Clark, and Clark T.-C. Nguyen, "High-Q HF Microelectromechanical Filters," IEEE J. Solid-State Circuits, vol. 35, NO. 4, pp. 512-526, Apr. 2000.

[3] A. A. Abidi, "Direct-conversion radio transceivers for digital communications," IEEE J. Solid-State Circuits, vol. 30, pp. 1399-1410, Dec. 1995.

[4] S.Timoshenko et al., Vibration Problems in Engineering, 1974.

[5] H. A. C. Tilmans, J. "Equivalent circuit representation of electromechanical Lumped-parameter systems," Micromech. Microeng, pp. 157-176, 1996.

[6] A. I. Zverev, Handbook of Filter Synthesis, 1967.

[7] R. A. Johnson, Mechanical Filters in Electronics, Wiley, 1983.

[8] M. Al_Khusheiny, B. Y. Majlis ,IEEE senior member, "Designing and Modeling a MEMS IF Bandpass Filter," International Conference on MEMS and Nanotechnology (ICMN'06), Kuala Lumpur, Malaysia.

[9] M. Al_Khusheiny \& B. Y. Majlis, senior member IEEE, "Optimization Design of Clamped-Clamped Resonator, " NSM 2005 Conference, Kuching, Malaysia, ,pp. 215-220,Nov. 22-24, 2005.

\section{Conclusions}

